\title{
Intraosseous Odontoma
}

\author{
${ }^{1}$ Geeta Singh, ${ }^{2}$ Shadab Mohammad, ${ }^{3}$ Laxman R Malkunje, ${ }^{3}$ Nimisha Singh \\ ${ }^{1}$ Assistant Professor, Department of Oral and Maxillofacial Surgery, CSM Medical University, Lucknow, Uttar Pradesh, India \\ ${ }^{2}$ P rofessor and Head, Department of Oral and Maxillofacial Surgery, CSM Medical University, Lucknow, Uttar Pradesh, India \\ ${ }^{3}$ P ostgraduate Student, Department of Oral and Maxillofacial Surgery, CSM Medical University, Lucknow, Uttar Pradesh, India
}

Correspondence: Geeta Singh, Assistant Professor, Department of Oral and Maxillofacial Surgery, CSM Medical University Lucknow-226003, Uttar Pradesh, India, Phone: 09415109713, e-mail: drgeetasinghkgmc@ gmail.com

\section{ABSTRACT}

Odontomes show no gender predilection, and are most often diagnosed in the second decade of life. They are preferentially located in the upper maxilla, particularly in the anterior sector. Compound odontome are more prevalent than complex odontome, and show no predilection in terms of patient gender, age or location. We hereby report a case of large compound odontome in the mandibular angle region of a 55year-old woman.

Most such lesions are asymptomatic and constitute casual findings in X-ray studies indicated for other reasons. The most common clinical manifestations are absence of impacted teeth and the presence of a tumor. Treatment consists of surgical removal of the lesion. The prognosis is very good, with a scant tendency towards relapse.

Keywords: Compound odontome, Intraosseous odontome, Rare case.

\section{INTRODUCTION}

Odontomas are nonaggressive, hamartomatous developmental mal formations or lesions of odontogenic origin, which consist of enamel, dentin, cementum and pulpal tissue (hence, they are also called composite, i.e. consisting of multiple or more than one type of tissue) and constitute $22 \%$ of all odontogenic tumors. ${ }^{1}$ T wo types of odontomas are recognized-compound and complex. Compound odontomas consist of encapsulated, discrete, small tooth-like structures or denticles in a fibrous connective tissue stroma. Complex odontomas on the other hand, consist of an irregular mixture or mass of mature hard and soft dental tissues which are poorly differentiated as enamel, dentin or cementum, and hence have no resemblance to teeth. ${ }^{2}$ Clinically, three types of odontomas are described: Central (intraosseous) odontomas, peripheral (extraosseous or soft tissue) odontomas and erupted odontomas.

Rarely, intraosseous odontomas located coronally to an erupting or impacted tooth or superficially in bone may facilitate their eruption into the oral cavity. These lesions have traditionally been referred to as erupted odontomas.

The etiology of odontomas is unknown but genetic factors and environmental causes, such as trauma and infection, have been proposed. M ost odontomas discovered during the first two decades of life and there is no significant gender predilection. They often remain asymptomatic and undiscovered until revealed by routine radiography where they characteristically appear as dense, radiopaque masses. ${ }^{3}$ Sometimes clinical indicators of their presence may include bone expansion, pain and tooth displacement or unerupted normal teeth. Enucleation is curative and recurrence is not a problem.
Radiological examination showed absence of mandibular third molars. A radiopaque lesion was seen posterior to first molar tooth, measuring about $2 \times 3 \mathrm{~cm}$ in greatest dimension.

We report a case of intraosseous odontoma in a 55 -year-old female. With a large bony swelling in the angle region of mandible, which was managed surgically.

\section{CASE REPORT}

A 55-year-old female presented to our department with pain and swelling at the left side of the angle region of mandible for the past one year. Extraoral examination revealed a single, localized, tender, and bony hard swelling (approx. $2 \times 3 \mathrm{~cm}$ ) at left side of angle region of mandible along with sinus present $2 \mathrm{~cm}$ below the lower border of mandible (Fig. 1). The teeth were vital and asymptomatic with no carious, periapical or

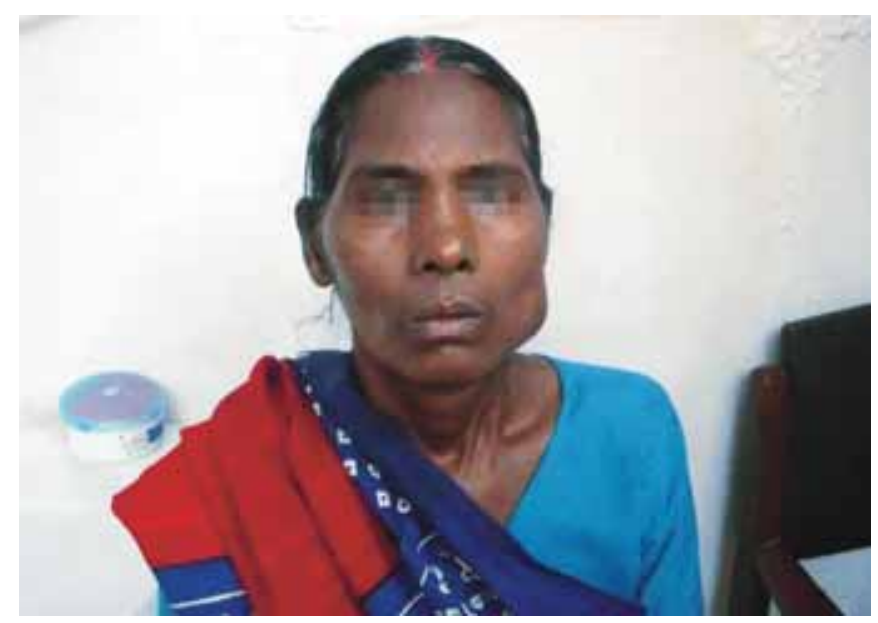

Fig.1: Preoperative photograph 


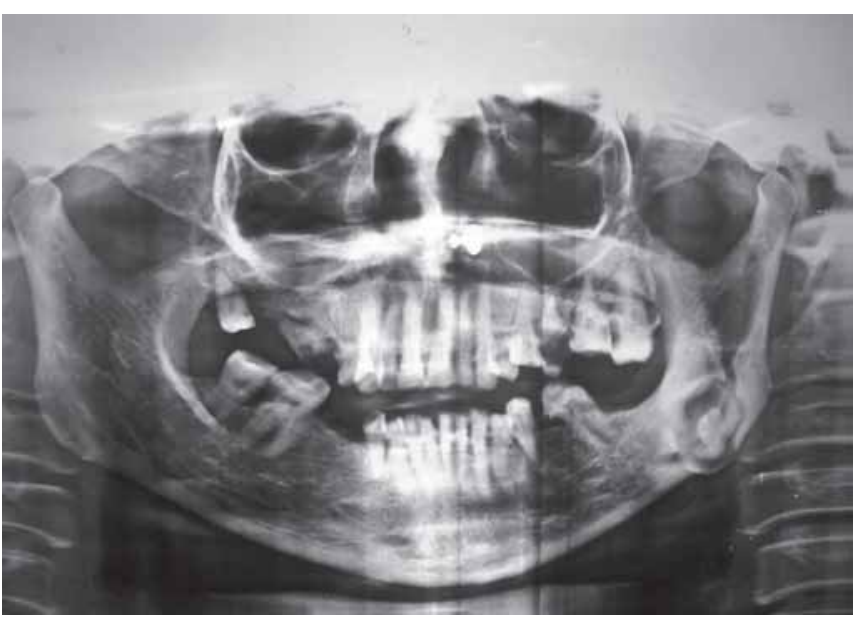

Fig. 2: Orthopantomogram revealing the lesion

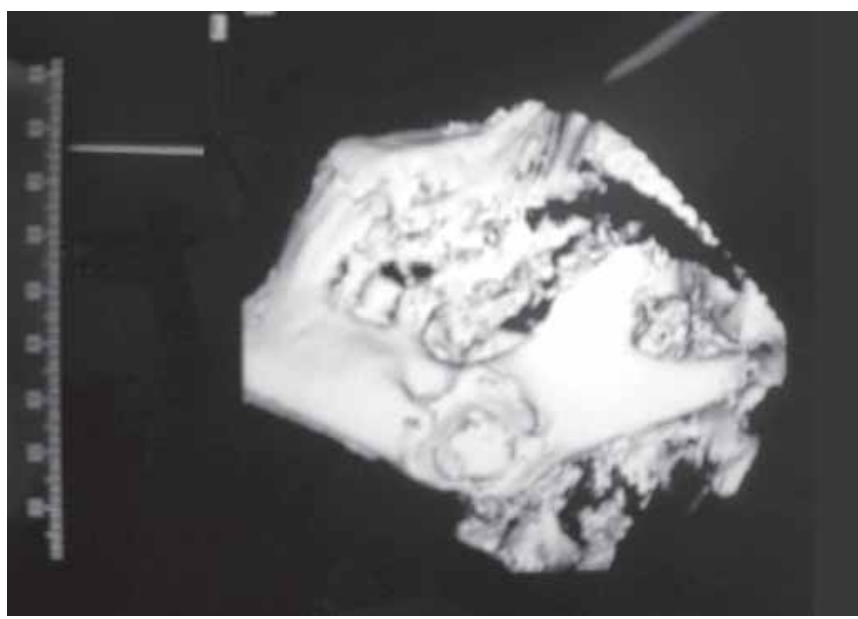

Fig. 3: Dentascan showing intraosseous lesion

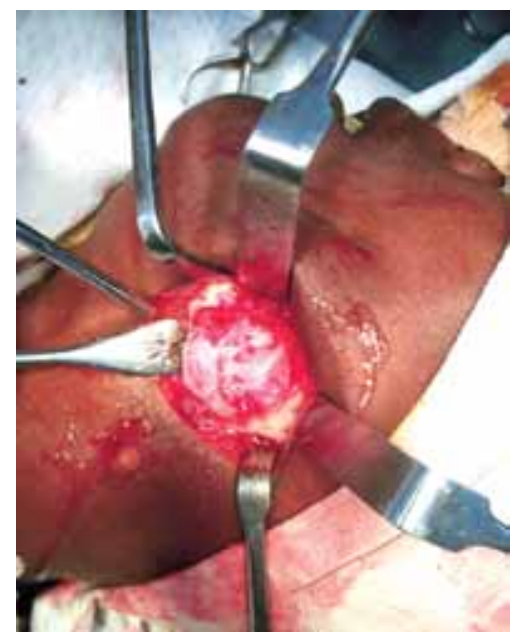

Fig. 4: Intraoperative photograph

periodontal involvement. The patient was healthy with an unremarkable medical history.

Radiograph included orthopantomogram (Fig. 2) and dentascan (Fig. 3) panoramic view.

Radiograph revealed a radiopaque mass in the left angle region of mandible measuring about $3 \times 2.5 \mathrm{~cm}$ with large ovoid

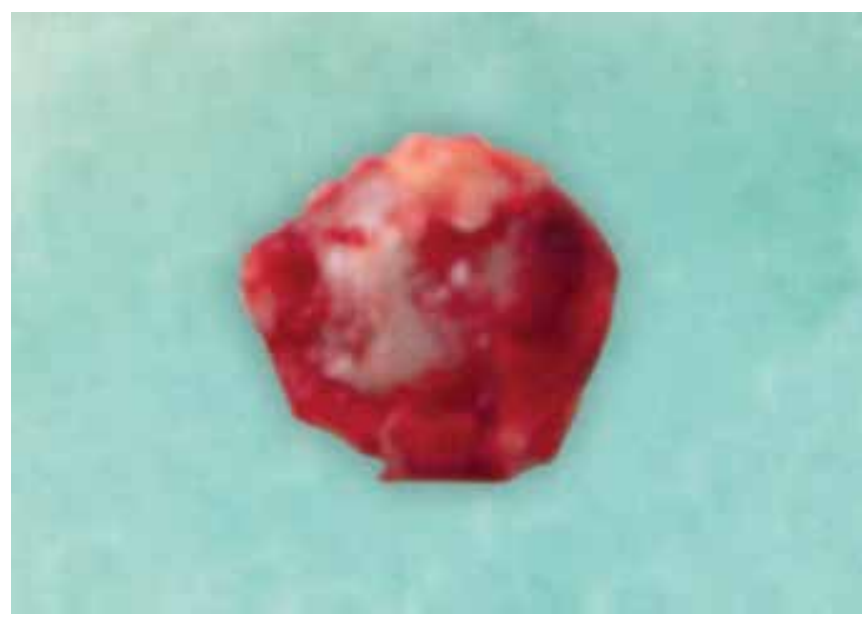

Fig. 5: Intraoperative entire lesion

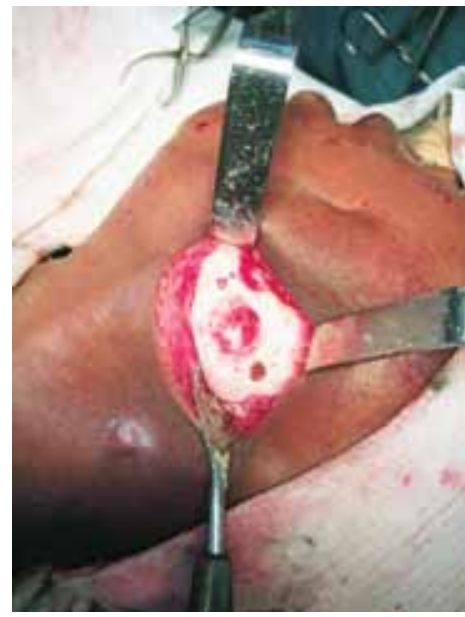

Fig. 6: Odontome after removal

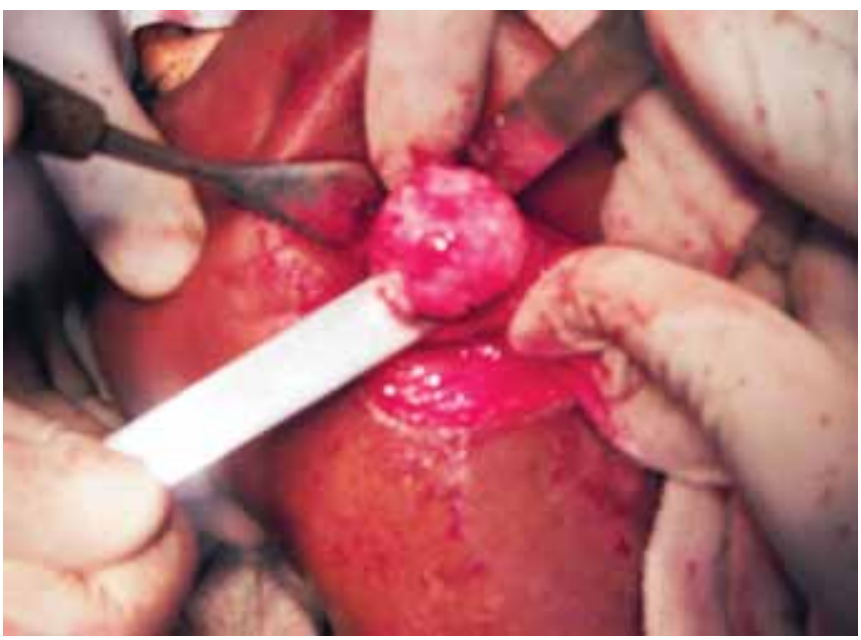

Fig. 7: Bony cavity after surgical removal of odontome

well-defined border and radiolucent lining with the angle of mandible with missing 2nd and 3rd molar teeth.

The differential diagnosis of the radiopacity included periapical cemental dysplasia and benign cementoblastoma.

Lesion was provisionally diagnosed as odontomas. Surgery was planned under general anesthesia after routine 
hematological investigations. The submandibular incision was given and layer wise dissection was done and the lesion was exposed and taken out (Figs 4 to 7). The flap was repositioned and sutured. The histopathological report confirmed the diagnosis of complex odontoma.

\section{DISCUSSION}

Odontomas are the most common odontogenic tumor. They are considered to be hamartomas rather than neoplasms, and are composed of the tissues native to teeth: Enamel, dentin, cementum and pulp tissue. 0 dontomas are further subclassified based upon their gross and radiographic features into compound (small tooth-like structures) or complex (a conglomeration of dentin, enamel and cementum). ${ }^{4}$

Odontomas may be found at any age; however, most are detected in the first two decades of life. There is no gender predilection and most lesions are detected on routine radiographs. Clinical symptoms are uncommon, however, an affected patient may present when a permanent tooth or multiple teeth that fail to erupt. ${ }^{5} \mathrm{R}$ arely, an odontoma may erupt into the oral cavity. ${ }^{6}$ Compound odontomas are more commonly found in the anterior maxilla, while complex odontomas tend to favor the posterior mandible. Odontomas are generally small; however, they may occasionally grow large resulting in bone expansion. ${ }^{4}$ Although odontomas may be found in any toothbearing region of the jaws, majority of the complex odontomas are found in the posterior mandible, followed by the anterior maxilla and is usually discovered before the second decade of life. ${ }^{7}$

$\mathrm{Histologically,} \mathrm{the} \mathrm{odontoma} \mathrm{is} \mathrm{not} \mathrm{a} \mathrm{diagnostic} \mathrm{dilemma.}$ It is composed of dentin, cementum, pulpal tissue and enamel. However, mature enamel is lost during the decalcification processing and will not be seen on conventional hematoxylin and eosin stained slides. The compound odontoma recapitulates the organization of a normal tooth, while the complex odontoma appears as a disorganized mass of hard odontogenic tissues. ${ }^{1}$ Loose, myxoid connective tissue with odontogenic epithelial rests may be seen in close association with the lesion, and most often represents normal dental follicular tissue. Fibrous connective tissue with a cystic lining representing a dentigerous cyst may also be seen. Treatment for an odontoma is complete removal with any associated soft tissues. Recurrences do not occur. Orthodontic treatment may be indicated to correct any malocclusion.

The etiology of odontomas has been attributed to various pathological conditions, like local trauma, inflammatory or infectious process, hereditary anomalies (Gardner's syndrome, Harmann syndrome), odontoblastic hyperactivity and alternation in the genetic component responsible for controlling dental development.

There is no consensus regarding gender predilection. Some studies have reported a greater incidence in femal es while other report a higher incidence in males and some studies report no differences between males and females in coincidence with the results of our own group of patients. R eview done by slight male predilection for compound odontomas was observed, while complex odontomas were slightly more common in females.

\section{REFERENCES}

1. Wood NK, Goaz PW, L ehnert JF. Differential diagnosis of oral and maxillofacial lesions. St Louis: M osby 1997:415-32.

2. White SC, Pharoah MJ. Oral Radiology principles and interpretation. St L ouis: M osby 2004:410-56.

3. Regezi JA, Sciubba JJ. Oral pathology: Clinical pathologic correlations (3rd ed). Philadelphia: Saunders 1999:323-56.

4. Bordini J J r, Contar CM , Sarot JR, Fernandes A, M achado M A. $M$ ultiple compound odontomas in the jaw: Case report and analysis of the literature. J Oral Maxillofac Surg 2008;66(12):2617-20.

5. Nagaraj K, U padhyay M, Y adav S. I mpacted maxillary central incisor, canine, and second molar with 2 supernumerary teeth and an odontoma. Am J Orthod Dentofacial Orthop 2009;135(3):390-99.

6. Serra-Serra G, Berini-A yte's L, Gay-Escoda C. Erupted odontomas: A report of three cases and review of the literature. Med Oral Patol Oral Cir Bucal 2009;14(6):E299-303.

7. Tomizawa M, Otsuka $Y$, Noda T. Clinical observations of odontomas in J apanese children: 39 cases including one recurrent case. Int J Paedtr Dent 2005;1:37-43. 